\title{
Scenario-based Risk Management and Simulation Optimization
}

Contributed to the Wiley Encyclopedia of Quantitative Risk Assessment

$$
\text { By: }
$$

\section{Marco Better}

OptTek Systems, Inc.

Address: 2241 17th Street

Boulder, CO 80302

Phone: (303) 447-3255 x. 109

Fax: (303) 447-3886

Email: better@colorado.edu

\section{Fred Glover}

OptTek Systems, Inc.

Address: 2241 17th Street

Boulder, CO 80302

Phone: (303) 447-3255 x. 106

Fax: (303) 447-3886

Email: glover@opttek.com

\section{Contents:}

1. Article title

2. Contributor name(s)

3. Basic contributor affiliation(s)

4. Keywords

5. Abstract

6. Introduction

7. Main text

8. References

9. Figure captions

10. Figures 


\title{
Scenario-based Risk Management and Simulation Optimization
}

Marco Better

OptTek Systems, Inc., 1919 Seventh Street,Boulder,CO 80302,USA, better@,colorado.edu.

\section{Fred Glover}

OptTek Systems, Inc., 1919 Seventh Street,Boulder, CO 80302,USA,glover@opttek.com.

\begin{abstract}
Traditional optimization approaches for handling uncertainty and risk typically require severe assumptions that are often not satisfied in complex practical settings. In an effort to overcome such limitations, several methods have been developed to handle uncertainty when the data and associated real world parameters do not behave according to classical assumptions. Two of the leading and most widely used examples are scenario optimization and robust optimization, both of which seek high-quality decisions that are feasible for all scenarios. However, both of these approaches likewise succumb to deficiencies encountered by classical methods, by exhibiting serious limitations in terms of the complexity and size of the models they can handle. Simulation optimization overcomes these limitations, and its flexibility and ease of use has contributed to its popularity as a preferred optimization approach to risk management applications.
\end{abstract}

Key Terms: $\quad$ optimization, simulation, robust optimization, scenario optimization, portfolio selection, uncertainty, risk management 


\section{INTRODUCTION}

Without uncertainty there is no risk. However, very few - if any - real-world situations are riskfree. In the world of deterministic optimization, we often choose to "ignore" uncertainty in a given situation in order to come up with a unique and objective solution to a problem. But in situations where uncertainty is at the core of the problem, as it is in those which involve risk management, a different strategy is required.

In the field of optimization, there are various approaches designed to cope with uncertainty. In this context, the exact values that the parameters (e.g. the data) of the optimization problem will have are not known with absolute certainty, but may vary to a larger or lesser extent depending on the nature of the factors they represent. In other words, there may be many possible "realizations" of the parameters, each of which is called a scenario.

Sections 1 and 2 briefly describe traditional scenario-based approaches to optimization, such as scenario optimization and robust optimization. Section 3 then delves in greater detail into a recently emerging approach that is having a profound impact on risk management called simulation optimization.

\section{SCENARIO OPTIMIZATION}

Dembo in [1] offers an approach to solving stochastic programs based on a method for solving deterministic scenario subproblems and combining the optimal scenario solutions into a single feasible decision.

Imagine a situation in which we want to minimize the cost of producing a set $J$ of finished goods. Each good $j(j=1, \ldots, n)$ has a per-unit production cost $c_{j}$ associated with it, as well as an associated utilization rate $a_{i j}$ of resources for each finished good. In addition, the plant that produces the goods has a limited amount of each resource $i(i=1, \ldots, m)$, denoted by $b_{i}$. We 
can formulate a deterministic mathematical program for a single scenario $s$ (the scenario subproblem, or $S P$ ) as follows:

$$
\begin{array}{ll}
\underline{\text { SP: }} & \\
z^{s}= & \min \sum_{j=1}^{n} c_{j}^{s} x_{j} \\
\text { Subject to: } & \sum_{j=1}^{n} a_{i j}^{s} x_{j}=b_{i}^{s} \quad \text { for } i=1, \ldots, m \\
& x_{j} \geq 0 \quad \text { for } j=1, \ldots, n
\end{array}
$$

where $c^{s}, a^{s}$ and $b^{s}$ respectively represent the realization of the cost coefficient, the resource utilization and the resource availability data under scenario $s$. Consider, for example, a company that a certain type of Maple door. Depending on the weather in the region where the wood is obtained, the costs of raw materials and transportation will vary. The company is also considering whether to expand production capacity at the facility where doors are manufactured,

\begin{tabular}{|c|c|c|c|c|c|c|c|c|}
\hline Scenario & Capacity & $\mathbf{P}(\mathrm{C})$ & Weather & $\mathbf{P}(\mathrm{W})$ & P(Scenario) & $\begin{array}{c}\text { Cost } \\
c_{i}\end{array}$ & $\begin{array}{c}\text { Utilization } \\
a_{i j}\end{array}$ & $\begin{array}{l}\text { Availability } \\
\qquad b_{i}\end{array}$ \\
\hline 1 & \multirow{3}{*}{ Current } & \multirow{3}{*}{$50 \%$} & Dry & $33 \%$ & $1 / 6$ & Low & High & Low \\
\hline 2 & & & Normal & $33 \%$ & $1 / 6$ & Medium & Low & Low \\
\hline 3 & & & Wet & $33 \%$ & $1 / 6$ & High & Low & Low \\
\hline 4 & \multirow{3}{*}{ Expanded } & \multirow{3}{*}{$50 \%$} & Dry & $33 \%$ & $1 / 6$ & Low & High & High \\
\hline 5 & & & Normal & $33 \%$ & $1 / 6$ & Medium & Low & High \\
\hline 6 & & & Wet & $33 \%$ & $1 / 6$ & High & Low & High \\
\hline
\end{tabular}
so that a total of six scenarios per door type must be considered. The six possible scenarios and associated parameters for Maple doors are shown in Table 1.

Table 1: Possible Scenarios for Maple Doors

Therefore, model SP needs to be solved once for each of the six scenarios.

The scenario optimization approach can be summarized in two steps:

1. Compute the optimal solution to each deterministic scenario subproblem $S P$.

2. Solve a tracking model to find a single, feasible decision for all scenarios. 
The key aspect of scenario optimization is the function of the tracking model in step 2. For illustration we introduce a simple form of tracking model. Let $p^{s}$ denote the estimated probability for the occurrence of scenario $s$. Then, a simple tracking model for our problem can be formulated as follows:

$$
\begin{aligned}
& \text { Minimize } \quad \sum_{s} p^{s}\left(\sum_{j} c_{j}^{s} x_{j}-z^{s}\right)^{2}+\sum_{s} p^{s}\left(\sum_{i j} a_{i j}^{s} x_{j}-b_{i}^{s}\right)^{2} \\
& \text { Subject to: } \quad x_{j} \geq 0 \quad \text { for } j=1, \ldots, n
\end{aligned}
$$

The purpose of this tracking model is to find a solution that is feasible under all the scenarios, and penalizes solutions that differ greatly from the optimal solution under each scenario. The two terms in the objective function are squared to ensure non-negativity. More sophisticated tracking models can be used for various different purposes. In risk management, for instance, we may select a tracking model that is designed to penalize performance below a certain target level.

\section{ROBUST OPTIMIZATION}

Robust optimization may be used when the parameters of the optimization problem are known only within a finite set of values. The robust optimization framework gets its name because it seeks to identify a robust decision - i.e. a solution that performs well across many possible scenarios.

In order to measure the robustness of a given solution, different criteria may be used. Kouvelis and Yu identify three criteria: (1) Absolute robustness; (2) Robust deviation; and (3) Relative robustness. We illustrate the meaning and relevance of these criteria, by describing the robust optimization approach described in [2].

Consider an optimization problem where the objective is to minimize a certain performance measure such as cost. Let $S$ denote the set of possible data scenarios over the 
planning horizon of interest. Also, let $X$ denote the set of decision variables, and $P$ the set of input parameters of our decision model. Correspondingly, we let $P^{s}$ identify the value of the parameters belonging to scenario $s$, and let $F^{s}$ identify the set of feasible solutions to scenario $s$. The optimal solution to a specific scenario $s$ is then:

$$
z^{s}=f\left(X_{s}^{*}, P^{s}\right)=\min _{X \in F^{s}} f\left(X, P^{s}\right)
$$

We assume here that $f$ is convex. The first criterion, absolute robustness, also known as "worst-case optimization," seeks to find a solution that is feasible for all possible scenarios and optimal for the worst possible scenario. In other words, in a situation where the goal is to minimize the cost, the optimization procedure will seek the robust solution, $z^{R}$, that minimizes the cost of the maximum-cost scenario. We can formulate this as an objective function of the form

$$
z^{R}=\min \left\{\max _{s \in S} f\left(X, P^{s}\right)\right\}
$$

The second criterion, robust deviation, is the one that minimizes the largest deviation from optimality. In other words, we seek to minimize the worst-case deviation that separates the robust solution from the optimal solution to each scenario. The third criterion, relative robustness, is similar to the second, but instead of a direct deviation measure, we use a relative deviation measure with respect to optimality. Suppose for convenience we reformulate the scenario subproblem introduced in the previous section as follows(based on [2], pp. 26-29):

$$
z^{s}=\quad \min c^{s} x
$$

$$
\begin{array}{ll}
\text { Subject to: } & A^{s} x=b^{s} \\
& x \geq 0
\end{array}
$$


Then, the robust deviation problem would be formulated as:

$$
\begin{array}{ll}
\text { Minimize } & y \\
\text { Subject to: } & c^{s} x \leq y+z^{s} \\
& A^{s} x=b^{s} \\
& x \geq 0
\end{array}
$$

Variations to this basic framework have been proposed in [3] to capture the risk-averse nature of decision-makers, by introducing higher moments of the distribution of $z^{s}$ in the optimization model, and implementing weights as penalty factors for infeasibility of the robust solution with respect to certain scenarios.

\section{SIMULATION OPTIMIZATION}

Until quite recently, the methods available for finding optimal decisions have been unable to effectively handle the complexities and uncertainties posed by many real world situations of the form treated by simulation. The areas of scenario optimization and robust optimization described in the previous sections attempt to deal with some of these, but the modeling framework limits the range of situations that can be tackled with such technology.

The complexities and uncertainties that render real world systems mathematically intractable are the primary reason that simulation is often chosen as a basis for handling decision problems associated with those systems. On one hand, the set of possible scenarios is often unknown a priori. On the other hand, the possible combinations of parameters is too numerous to be handled efficiently by methods such as those previously described. Consequently, decision makers must deal with the dilemma that many important types of optimization problems can only be treated by the use of simulation models, but once these problems are submitted to simulation there are no optimization methods that can adequately cope with them. 
Recent developments are changing this picture. The field of metaheuristics - the domain of optimization that augments traditional mathematics with artificial intelligence and methods derived from physical, biological or evolutionary analogs - has witnessed advances yielding effective engines for guiding a series of complex evaluations in the quest for optimal values for the decision variables, as described in [4, 5, 6, 7, 8 and 9].

Modern simulation optimization tools are designed to solve optimization problems of the form

$$
\begin{array}{lll}
\text { Minimize } & F(x) & \text { (Objective function) } \\
\text { Subject to: } & A x \leq b & \text { (Constraints) } \\
& g_{l} \leq G(x) \leq g_{u} \text { (Requirements) } \\
& l \leq x \leq u \quad \text { (Bounds) }
\end{array}
$$

where the vector $x$ of decision variables includes variables that range over continuous values and variables that only take on discrete values (both integer values and values with arbitrary step sizes).

The objective function $F(x)$ is typically highly complex; under the context of simulation optimization $F(x)$ represents a performance measure output by the simulation, and may be any mapping from a set of values $x$ to a real value. The constraints represented by inequality $A x \leq b$ are linear (given that non-linearity in the model is embedded within the simulation itself), and the coefficient matrix $A$ and the right-hand-side values corresponding to vector $b$ must be known. The requirements $g_{l} \leq G(x) \leq g_{u}$ impose simple upper and/or lower bounds on a function that can be linear or non-linear, and is an output of the simulation. The values of the bounds $g_{l}$ and $g_{u}$ must be known constants. All the variables are bounded and some may be restricted to be 
discrete, as previously noted. Each evaluation of $F(x)$ and $G(x)$ requires a simulation of the system. By combining simulation and optimization, a powerful design tool results.

Optimizers designed for simulation embody the principle of separating the method from the model. In such a context, the optimization problem is defined outside the complex system. Therefore, the evaluator (i.e. the simulation model) can change and evolve to incorporate additional elements of the complex system, while the optimization routines remain the same. Hence, there is a complete separation between the model that represents the system and the procedure that is used to solve optimization problems defined within this model.

The optimization procedure uses the outputs from the system evaluator, which measures the merit of the inputs that were fed into the model. On the basis of both current and past evaluations, the method decides upon a new set of input values (see Figure 1).

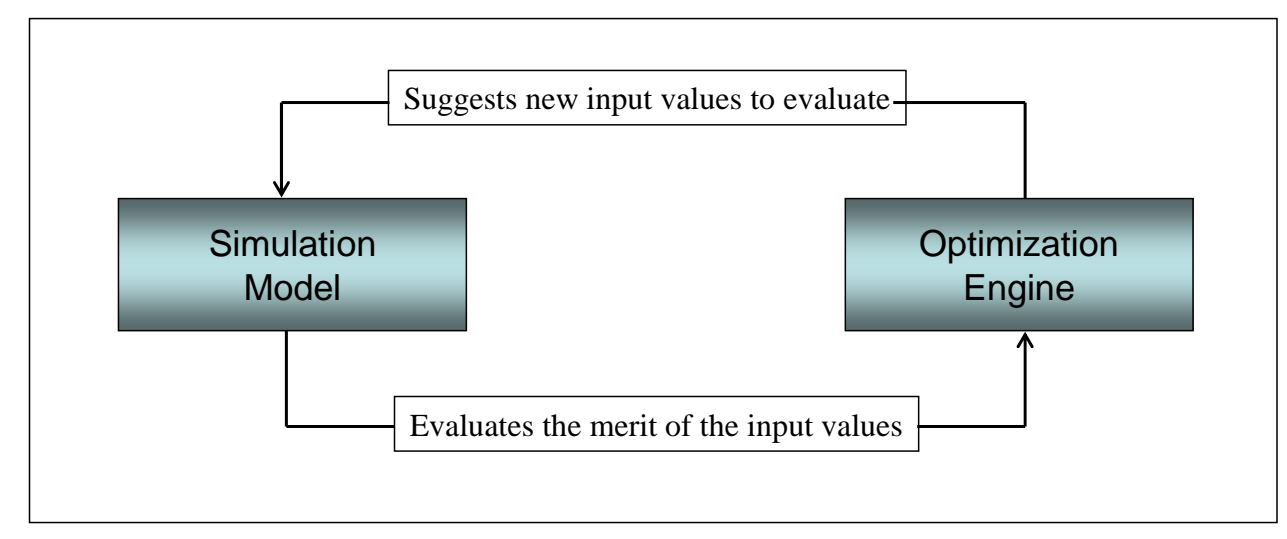

Figure 1: Coordination between Optimizer and Simulator

Provided that a feasible solution exists, the optimization procedure ideally carries out a special search where the successively generated inputs produce varying evaluations, not all of them improving, but which over time provide a highly efficient trajectory to the globally best solutions. The process continues until an appropriate termination criterion is satisfied (usually based on the user's preference for the amount of time devoted to the search). 
The simulation optimization framework is very flexible in terms of the performance measures the decision-maker wishes to evaluate. In fact, the only limitation is not on the side of the optimization engine, but on the simulation model's ability to evaluate performance based on specified values for the decision variables. In order to provide in-depth insights into the use of simulation optimization in the context of risk-management, we present a practical application through the use of an illustrative example in the context of optimal portfolio selection.

\section{Example: Selecting Optimal Portfolios of Projects}

The Energy Industry uses project portfolio optimization to manage investments in exploration and production, as well as power plant acquisitions $[10,11]$. Decision makers typically wish to maximize the return on invested capital, while controlling the exposure of their portfolio of projects to various risk factors.

The following example involves a company that has sixty-one potential projects in its investment funnel. Each type of project requires an initial investment and a certain number of business development, engineering and earth sciences personnel. The company has a budget limit for its investment opportunities, and a limited number of personnel of each skill category. Projects may start in different time periods, but there is a restricted window of opportunity of up to three years for each project. The company must select a set of projects to invest in that will best further its corporate goals.

Probably the best-known model for portfolio optimization is rooted in the work of Nobel laureate Harry Markowitz. The model, called the mean-variance model [12], is based on the assumption that the expected portfolio returns will be normally distributed. The model seeks to balance risk and return in a single objective function, as follows. 
Given a vector of portfolio returns $r$, and a covariance matrix $Q$ of returns, then we can formulate the model as follows:

$$
\text { Maximize } \quad r^{T} w-k w^{T} Q w
$$

Subject to:

$$
\sum_{\mathrm{i}} c_{i} w_{i}=b
$$

$$
w \in\{0,1\}
$$

where $k$ represents a coefficient of the firm's risk aversion, $c_{i}$ represents the initial investment in project $i, w_{i}$ is a binary variable representing the decision whether to invest in project $i$, and $b$ is the available budget. We will use the mean-variance model as a base case for the purpose of comparing to other selected models of portfolio performance.

To facilitate our analysis, we make use of the OptFolio ${ }^{\circledR}$ software that combines simulation and optimization into a single system specifically designed for portfolio optimization [13, 14 and 15].

We examine three cases to demonstrate the flexibility of this method to enable a variety of decision alternatives that significantly improve upon traditional mean-variance portfolio optimization, and illustrate the flexibility afforded by simulation optimization approaches in terms of controlling risk. The results also show the benefits of managing and efficiently allocating scarce resources like capital, personnel and time. The weighted average cost of capital, or annual discount rate, used for all cases was $12 \%$.

\section{Case 1: Mean-Variance Approach}

In this first case, we implement the mean-variance portfolio selection method of Markowitz described above. The decision is to determine participation levels ( 0 or 1 ) in each project with the objective of maximizing the expected net present value (NPV) of the portfolio while keeping 
the standard deviation of the NPV below a specified threshold of \$140M. We denote the expected value of the NPV by $\mu_{N P V}$, and the standard deviation of the NPV by $\sigma_{N P V}$. This case can be formulated as follows:

$\begin{array}{ll}\text { Maximize } & \mu_{N P V} \\ \sigma_{N P V}<\$ 140 M & \begin{array}{l}\text { (objective function)(18)Subject to: } \\ \sum_{i} c_{i} x_{i} \leq b\end{array} \\ \sum_{i} p_{i j} x_{i} \leq P_{j} \quad \forall j \quad \text { (requirement) } \\ \text { All projects must start in year } 1 \\ x_{i} \in\{0,1\} \quad \forall i \quad \text { (binary decisions) }\end{array}$

The optimal portfolio has the following performance metrics:

$$
\mu_{N P V}=\$ 394 \mathrm{M}, \sigma_{N P V}=\$ 107 \mathrm{M}, \mathrm{P}(5)_{\mathrm{NPV}}=\$ 176 \mathrm{M} \text {, }
$$

where $\mathrm{P}(5)_{\mathrm{NPV}}$ denotes the $5^{\text {th }}$ percentile of the resulting NPV probability distribution (i.e. the probability of the NPV being lower than the $\mathrm{P}(5)$ value is 5\%).

The bound imposed on the standard deviation in equation (19) does not seem binding. However, due to the binary nature of the decision variables, no project additions are possible without violating the bound. (See Figure 2.)

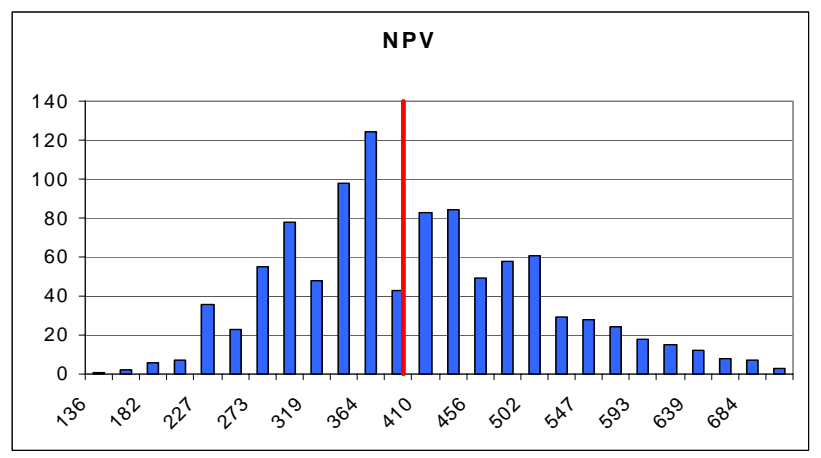

Figure 2: Mean-Variance Portfolio 
Figure 2 shows a graph of the probability distribution of the NPV obtained for 1000 replications of this base model. The thin line represents the expected value.

\section{Case 2: Risk controlled by $5^{\text {th }}$ Percentile}

In the context of risk management, statistics such as variance or standard deviation of returns are not always easy to interpret and other measures may be more intuitive and useful. For example, it is clearer to say: "there is a 95\% chance that the portfolio return is above some value X" than to say that the standard deviation is $\$ 107 \mathrm{M}$. This measure can easily be implemented in a simulation optimization procedure by imposing a requirement on the $5^{\text {th }}$ percentile of the resulting distribution of returns. In Case 2 , the decision is to determine participation levels $(0,1)$ in each project with the objective of maximizing the expected NPV of the portfolio, while keeping the $5^{\text {th }}$ percentile of the NPV distribution above the value determined in Case 1 . This is achieved by imposing the requirement in equation (25) on the model below. In other words, we want to find the portfolio that produces the maximum average return, as long as no more than 5\% of the observations fall below $\$ 176 \mathrm{M}$. Also, in this case we allow for delays in the start dates of projects, according to windows of opportunity defined for each project. In order to achieve this, we have created copies of a project that are shifted by one, two or three periods into the future (according to the windows of opportunity defined for each project). Mutual exclusivity clauses

ensure that only one start date for each project is selected. For example, to represent the fact that Project A can start at time $\mathrm{t}=0,1$ or 2 , we include the following mutual exclusivity clause as a constraint:

$$
\text { Project } A_{0}+\text { Project } A_{1}+\text { Project } A_{2} \leq 1
$$

The subscript following the project name corresponds to the allowed start dates for the project, and the constraint only allows at most one of these to be chosen. 


$$
\begin{aligned}
& \text { Maximize } \quad \mu_{N P V} \\
& \text { (24)Subject to:P(5) })_{N P V}>\$ 176 M \\
& \text { (requirement) } \\
& \sum_{i} c_{i} x_{i} \leq b \quad \text { (budget constraint) } \\
& \sum_{i} p_{i j} x_{i} \leq P_{j} \quad \forall j \quad \text { (personnel constraints) } \\
& \sum_{m_{i} \in M} x_{i} \leq 1 \quad \forall i \quad \text { (mutual exclusivity) } \\
& x_{i} \in\{0,1\} \forall I \quad \text { (binary decisions) }
\end{aligned}
$$

$m_{i}$ denotes the set of mutually exclusive projects related to project $i$.

where $m_{i}$ denotes the set of mutually exclusive projects related to project $i$.

In this case we have replaced the standard deviation with the $5^{\text {th }}$ percentile as a measure of risk containment. The resulting portfolio has the following attributes:

$$
\mu_{N P V}=\$ 438 \mathrm{M}, \sigma_{N P V}=\$ 140 \mathrm{M}, \mathrm{P}(5)_{\mathrm{NPV}}=\$ 241 \mathrm{M}
$$

By using the $5^{\text {th }}$ percentile instead of the standard deviation as a measure of risk, we are able to obtain an outcome that shifts the distribution of returns to the right, compared to Case 1 , as shown in Figure 3.

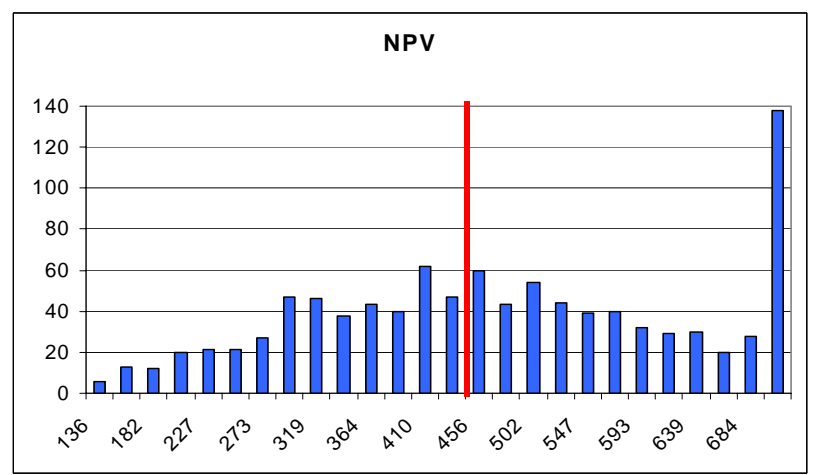

Figure 3: Portfolio Risk Controlled by P(5) 
This case clearly outperforms case 1 . Not only do we obtain significantly better financial performance, but we also achieve a higher personnel utilization rate, and a more diverse portfolio.

Case 3: Probability Maximizing and Value-at-Risk

In Case 3, the decision is to determine participation levels $(0,1)$ in each project with the objective of maximizing the probability of meeting or exceeding the mean NPV found in Case 1. This objective is expressed in equation (30) of the following model.

$$
\begin{aligned}
& \text { Maximize } \quad \boldsymbol{P}(\mathrm{NPV} \geq \$ 394 M) \\
& \text { Subject to: } \quad \sum_{i} c_{i} x_{i} \leq b \quad \text { (budget constraint) } \\
& \sum_{i} p_{i j} x_{i} \leq P_{j} \quad \forall j \quad \text { (personnel constraints) } \\
& \sum_{m_{i} \in M} x_{i} \leq 1 \quad \forall i \quad \text { (mutual exclusivity) } \\
& x_{i} \in\{0,1\} \quad \forall i \quad \text { (binary decisions) }
\end{aligned}
$$

This case focuses on maximizing the chance of achieving a goal and essentially combines performance and risk containment into one metric. The probability in (30) is not known a priori, so we must rely on the simulation to obtain it. The resulting optimal solution yields a portfolio that has the following attributes:

$$
\mu_{N P V}=\$ 440 \mathrm{M}, \sigma_{N P V}=\$ 167 \mathrm{M}, \mathrm{P}(5)=\$ 198 \mathrm{M}
$$

Although this portfolio has a performance similar to the one in Case 2, it has a 70\% chance of achieving or exceeding the NPV goal. As can be seen in the graph of Figure 4, we have succeeded in shifting the probability distribution even further to the right, therefore increasing our chances of exceeding the returns obtained with the traditional Markowitz 
approach. In addition, in cases 2 and 3, we need not make any assumption about the distribution of expected returns.

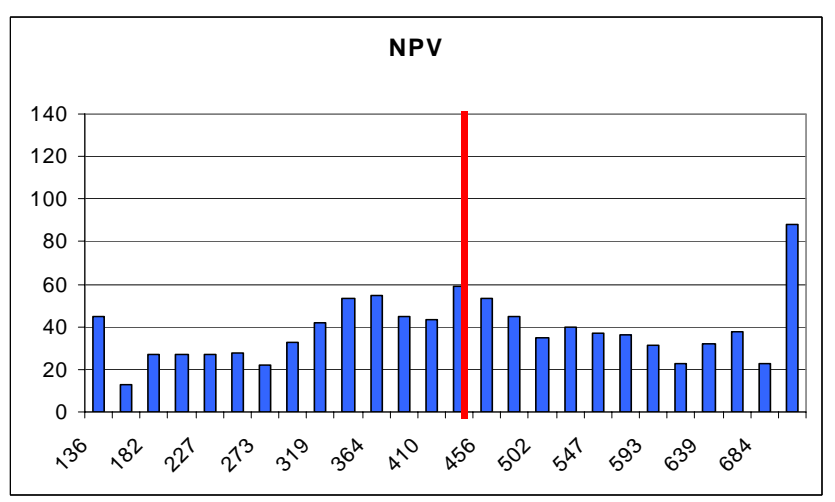

Figure 4: Probability-Maximizing Portfolio

As a related corollary to this last case, we can conduct an interesting analysis that addresses Value-at-Risk (VaR). In traditional (securities) portfolio management, VaR is defined as the worst expected loss under normal market conditions over a specific time interval and at a given confidence level. In other words, VaR measures how much the holder of the portfolio can lose with probability $=\alpha$ over a certain time horizon [16]. In the case of project portfolios, we can define VaR as the probability that the NPV of the portfolio will fall below a specified value. Going back to our present case, the manager may want to limit the probability of incurring negative returns. In this example, we formulate the problem in a slightly different way: we still want to maximize the expected return, but we limit the probability that we incur a loss to $\alpha=1 \%$ by using the requirement shown in equation (36) as follows:

Maximize $\quad \mu_{N P V}$

Subject to: $\quad \boldsymbol{P}(\mathbf{N P V}<0) \leq 1 \% \quad$ (requirement)

$$
\sum_{i} c_{i} x_{i} \leq b \quad \text { (budget constraint) }
$$




$$
\begin{array}{lll}
\sum_{i} p_{i j} x_{i} \leq P_{j} & \forall j & \text { (personnel constraints) } \\
\sum_{m_{i} \in M} x_{i} \leq 1 & \forall i & \text { (mutual exclusivity) } \\
x_{i} \in\{0,1\} & \forall i & \text { (binary decisions) }
\end{array}
$$

The portfolio performance under this scenario is:

$$
\mu_{N P V}=\$ 411 \mathrm{M}, \sigma_{N P V}=\$ 159 \mathrm{M}, \mathrm{P}(5)=\$ 195 \mathrm{M}
$$

These results from the VaR model turn out to be slightly inferior to the case where the probability was maximized. This is not a surprise, since the focus is on limiting the probability of downside risk, whereas before, the goal was to maximize the probability of obtaining a high expected return. However, this last analysis could prove valuable for a manager that wants to limit the VaR of the selected portfolio. As shown here, for this particular set of projects, a very good portfolio can be selected with that objective in mind.

\section{CONCLUSIONS}

Practically every real-world situation involves uncertainty and risk, creating a need for optimization methods that can handle uncertainty in model data and input parameters. We have briefly described two popular methods, scenario optimization and robust optimization, that seek to overcome limitations of classical optimization approaches for dealing with uncertainty, and which undertake to find high-quality solutions that are feasible under as many scenarios as possible. However, these methods are unable to handle problems involving moderately large numbers of decision variables and constraints, or involving significant degrees of uncertainty and complexity. In these cases, simulation optimization is becoming the method of choice. The combination of simulation and optimization affords all the flexibility of the simulation engine in terms of defining a variety of performance measures as desired by the decision maker. In 
addition, as we demonstrate through a project portfolio selection example, modern optimization engines can enforce requirements on one or more outputs from the simulation, a feature that scenario-based methods cannot handle. Finally, simulation optimization produces results that can be conveyed and grasped in an intuitive manner, providing an especially useful tool for identifying improved business decisions under risk and uncertainty.

\section{References}

1. Dembo R. Scenario Optimization. Annals of Operations Research 1991 30: 63-80.

2. Kouvelis P, Yu G. Robust Discrete Optimization and Its Applications; Kluwer: Dordrecht, Netherlands, 1997; pp. 8-29.

3. Mulvey JM, Vanderbei RJ, Zenios SA. Robust optimization of large-scale systems. Operations Research 1995 43.2: 264-281.

4. Campos V, Laguna M, Martí M. Scatter Search for the Linear Ordering Problem. New Methods in Optimization. McGraw-Hill: New York, NY, 1999; pp 331-339.

5. Glover F. A template for Scatter Search and Path Relinking. Artificial Evolution, Lecture Notes in Computer Science 1363. Springer-Verlag: New York, NY, 1998; pp. 13-54.

6. Glover F, Laguna M. Tabu Search. Kluwer: Norwell, MA, 1997.

7. Glover F, Laguna M, Martí R. Fundamentals of scatter search and path relinking. Control and Cybernetics 2000 29.3: 653-684.

8. Glover F, Laguna M, Martí R. Scatter Search, Advances in Evolutionary Computing: Theory and Applications. Springer-Verlag: New York, NY, 2003; pp 519-537.

9. Laguna M, Martí R. Scatter Search: Methodology and Implementations in C. Kluwer: Norwell, MA, 2003; pp 217-254. 
10. Haskett WJ. Optimal appraisal well location through efficient uncertainty reduction and value of information techniques. Proceedings of the Society of Petroleum Engineers Annual Technical Conference and Exhibition: Denver, CO 2003.

11. Haskett WJ, Better M, April J. Practical optimization: dealing with the realities of decision management. Proceedings of the Society of Petroleum Engineers Annual Technical Conference and Exhibition: Houston, TX 2004.

12. Markowitz H. Portfolio selection. Journal of Finance 1952 7.1: 77-91.

13. April J, Glover F, Kelly JP. Portfolio Optimization for Capital Investment Projects. Proceedings of the 2002 Winter Simulation Conference. pp. 1546-1554.

14. April J, Glover F, Kelly JP. Optfolio - A Simulation Optimization System for Project Portfolio Planning. Proceedings of the 2003 Winter Simulation Conference. pp. 301-309.

15. Kelly JP. Simulation Optimization is Evolving. INFORMS Journal of Computing 2002 14.3: $223-225$.

16. Benninga S, Wiener Z. Value-at-Risk (VaR). Mathematica in Education and Research 1998 7.4: $1-7$. 